\title{
Metastases of breast cancer causing acute appendicitis: A case report
}

\author{
Araújo JL ${ }^{1 *}$, Cavalcanti $\mathrm{BSM}^{2}$, Soares $\mathrm{MCDV}^{2}$, Sousa $\mathrm{UW}^{1}$ and Medeiros $\mathrm{GPD}^{3}$ \\ ${ }^{1}$ Department of Mammary Tumors, Liga Norte Rio-grandense contra o cancer, Natal, Brazil \\ ${ }^{2}$ School of Medicine, Potiguar University, Natal, Brazil \\ ${ }^{3}$ Resident of Surgery, Hospital Monsenhor Walfrdo Gurgel, Natal - RN, Brazil
}

\begin{abstract}
Background: Breast cancer (BC) is the most common type of cancer among women worldwide and advanced diagnosis is not uncommon. There are few data in the literature reporting the appearance of appendiceal and ovary metastases as the initial manifestation of primary neoplasia.

Objective: increase the knowledge about possible clinical presentations of BC with metastasis in the appendix and ovary and to discuss possible ways to prevent worse evolutions of the metastases under discussion.

Methods: This is a case report is a retrospective analysis of periodic consultations through the collection of data from the medical records obtained in the Liga NorteRiograndense Contra Câncer - Natal-RN (Brazil) of the patient under study between January 2014 to May 2018.

Case presentation: A 37-year-old woman presented abdominal pain with a mass in her right breast, near the areola, in addition there was a swollen lymph node in the right axilla and large pelvic mass. Given the severe abdominal pain, she was submitted to emergency exploratory laparotomy and in the intraoperative period were found inflammatory process characterized as acute appendicitis with blocked abscess, in addition to left ovary enlargement. During the investigation we discovered appendiceal and ovary metastases as the initial manifestation of $\mathrm{BC}$.
\end{abstract}

Conclusion: We report a relatively rare case of appendiceal and ovary metastases as the initial manifestation of BC with 52 months of no signs of illness. Although more studies are needed to explore targeted treatment to achieve better outcomes in this patient population.

\section{Introduction}

Breast cancer is the most common type of cancer among women worldwide, after non-melanoma skin, accounting for about $28 \%$ of new cases each year. Advanced diagnosis is not uncommon - less than $10 \%$ of patients with breast cancer (BC) have evidence of distant metastases at diagnosis [1]. It is well known that metastases induced by breast cancer are most commonly in the lungs, bones and liver. However, metastases of the appendix are extremely rare and acute appendicitis induced by a metastatic tumor is also uncommon [2]. Ovarian metastases is more common than the metastases of the appendix. Metastases and micrometastases in the ovaries have been reported with a prevalence ranging from $3 \%$ to $30 \%$ in various series, including autopsies, prophylactic or therapeutic oophorectomies, and incidental findings in routine surgery $[3,4]$.

In a review it was reported that incidental appendiceal tumor detected after appendectomy accounts for only $0.9 \%$ of cases (74/7990), and among them, metastatic tumor was only found in 11 cases [5], showing how extremely rare appendicular metastases cases are. The primary origins of metastatic appendix tumors are the breast, lung, stomach and colon [2]. The interval between the diagnosis of a primary cancer and the onset of appendicitis has varied from zero to six years $[2,6]$. There are few data in the literature reporting the appearance of appendiceal metastases as the initial manifestation of primary neoplasia.

The case that will be reported shows a rare diagnosis of breast cancer, with initial symptoms manifested by acute appendicitis due to appendicular metastasis, in addition to associated ovarian metastasis. The objective of this article is to increase the knowledge about possible clinical presentations of $\mathrm{BC}$ with metastasis in the appendix and ovary and to discuss possible ways to prevent worse evolutions of the metastases under discussion.

\section{Materials and methods}

This is a case report is a retrospective analysis of periodic consultations through the collection of data from the medical records, clinical, pathological and imaging studies obtained in the Liga NorteRiograndense Contra Câncer - Natal-RN (Brazil) of the patient under study. The period of analysis is from January 2014 to May 2018.

\section{Results}

A 37-year-old female patient, with no known risk factors for breast cancer, in December 2013 presented with severe abdominal pain. She showed a $4 \mathrm{~cm}$ diamenter mass in her right breast, near the areola, in addition there was a swollen lymph node in the right axilla and large

${ }^{\star}$ Correspondence to: Araújo JL, Department of Mammary Tumors, Liga Norte Rio-grandense contra o cancer, Natal, Brazil, E-mail: julopesaguiar@hotmail.com

Key words: breast cancer, ovarian metastases, appencieal metastases, appendicitis Received: May 13, 2018; Accepted: May 28, 2018; Published: May 31, 2018 
pelvic mass. Ultrasonography (US) of the pelvic region showed a large complex cistic in right region of pelvic with estimated volume of 552 $\mathrm{cm}^{3}$. The Breast's US presented in the right breast, a 1,6 cm diamenter mass, at 5 o'clock, near the areola and another mass with 2,6 cm diameter, at $6 \mathrm{o}^{\prime}$ clock, also near the areola, Birads 4 . Given the severe abdominal pain, she was submitted to emergency exploratory laparotomy. In the intraoperative period were found inflammatory process characterized as acute appendicitis with blocked abscess, in addition to left ovary enlargement. An appendectomy, hysterectomy with left oophorectomy and drainage of intrapelvic abscess were performed.

In January 2014 she was referred to the mastology service for investigation of the right breast nodule (RBN). During physical examination, a $5.5 \mathrm{~cm}$ diameter RBN was observed at 6 o'clock and another $3.3 \mathrm{~cm} \mathrm{RBN}$ at 12 o'clock, with retraction of the skin and negative axilla (Figure 1). The results of the histological examination and Immunohistochemistry of the left ovary and cecal appendix showed a poorly differentiated adenocarcinoma suggestive of metastases and was positive for cytokeratin 7, GCDPF15 and HER2 3+, negative for hormone receptors, respectively (Figure 2a-2c). She received six cycles of cyclophosphamide, fluorouracil and adriamycin therapy as neoadjuvant chemotherapy, and total mastectomy with axillary

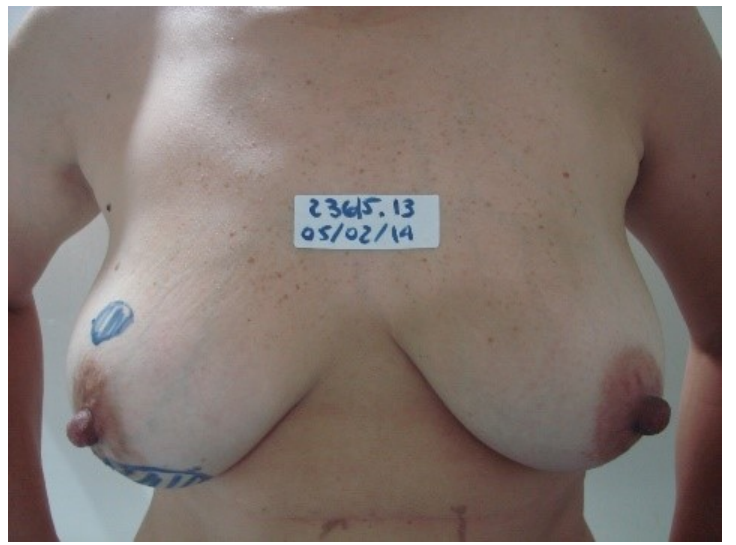

Figure 1: Images of mass in her right breast. $5.5 \mathrm{~cm}$ diameter RBN was observed at 6 o'clock and another $3.3 \mathrm{~cm} \mathrm{RBN}$ at 12 o'clock, with retraction of the skin and negative axilla

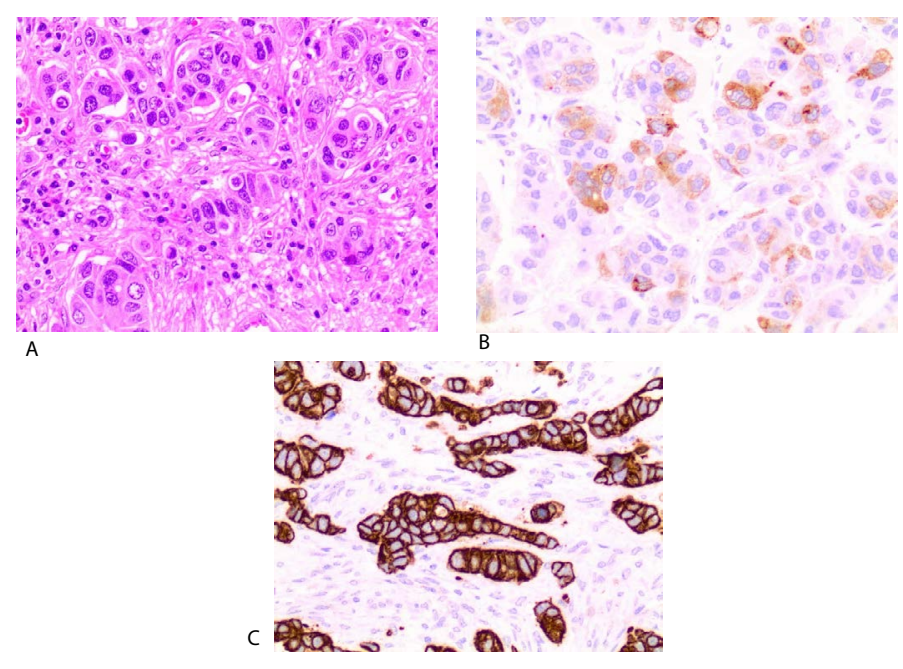

Figure 2: (A) Histopathological findings reveal small differentiated carcinoma showing solid and cordonary arrangements infiltrating ovarian parenchyma. (B,C) Immunohistochemical characterization expression of cytokeratin 7 and GCPDF15 in neoplastic nests dissection was performed in 2014. The results of the histological examination of the breast tumor and the axillary content showed a 2.5 $\mathrm{cm}$ diameter invasive ductal carcinoma, histologic grade 2 and nuclear grade 2 , and micrometastase in 3 of 11 lymph node.

By decision of the mastology and oncology's team of the Hospital Luis Antonio, she received six cicles of paclitaxel (adjuvant chemotherapy) and 28 cycles of adjuvant radiotherapy. Currently there are 52 months with no signs of illness. Perform periodic follow-up with multidisciplinary team of the Liga Norte-Riograndense Contra O Câncer.

\section{Discussion}

We present a case of acute appendicitis and ovarian tumor as initial findings of breast neoplasm, whose diagnosis was made through metastatic disease.

Acute metastases-induced appendicitis is uncommon with a few data in the world's literature. Connor et al. reported that incidental appendiceal tumor detected after appendectomy accounts for only $0.9 \%$ of cases (74/7990), and among them, metastatic tumor was only found in 11 cases [5]. Yoon et al. previously reported 139 cases with metastatic appendiceal tumors, with primary sites mainly consisting of ovary (56 cases), colon (35 cases), and stomach (7 cases) [7].

Ovarian metastases from breast cancer, although also of low prevalence, are more common than appendiceal metastase. Metastases and micrometastases in the ovaries have been reported with a prevalence ranging from $3 \%$ to $30 \%$ in various series, including autopsies, prophylactic or therapeutic oophorectomies, and incidental findings in routine surgery $[3,4]$. Approximately $6 \%-7 \%$ of ovarian cancers and $10 \%$ of bilateral ovarian malignancies discovered during surgical intervention are metastatic [8,9]. Although invasive lobular carcinoma has a much greater tendency to metastasize to the ovary, $75 \%$ of ovarian metastases are from invasive ductal cancers due to its higher prevalence [10].

Bigorie et al. in a retrospective analysis of 29 cases, found a predominance of premenopausal status, receptor-positive hormone, a higher prevalence of infiltrating lobular carcinoma, bilateral breast cancer, and predisposing genetic factors compared to the world population with breast cancer [11]. The diagnosis of ovarian metastases was given in an average of 5 years after the discovery of the primary tumor (1-19 years [10]). To date, there has been no systematic review involving ovarian metastasis and positive hormone receptors or premenopausal status, which generates only the hypothesis of hormonal regulation in this subgroup of patients.

There is no literature finding of acute appendicitis as the initial finding of breast cancer, triggered by appendicular and ovarian metastases. The patient's age is below the average found in the cases already reported. The prognosis of patients with appendix metastasis is not good, especially when it opens with appendicitis. The median survival after a diagnosis of a secondary appendiceal tumors was 22.6 months [12]. The likely reason for such poor prognosis is that isolated metastasis to the appendix is rare, and peritoneal metastases were frequently detected in the previous patients [12]. Our patient has already 52 months of life with no sign of new metastatic sites or recurrence of the disease.

Although that some groups have even advocated for prophylactic appendectomy at time of oophorectomy or other abdominal surgery in the breast cancer population [13], we do not yet have formal studies with protocols that indicate a prophylactic appendectomy in 
these patients. The conduct of ovarian metastasis follows the same principle. Recent studies have indicated a possible survival benefit after cytoreductive surgery; however, the criteria for patient selection remain obscure [11]. The high rates of hormone receptor positive tumors and premenopausal patients led the authors to suggest that the surgical option should consist of at least bilateral oophorectomy, even when the contralateral ovary appears normal [11].

\section{Conclusion}

More studies are needed to explore targeted treatment to achieve better outcomes in this patient population. Although metastases to the appendix causing acute appendicitis are rare in clinical practice, this should be considered as part of the differential diagnosis when a cancer patient presents with suggestive symptoms [2]. Special attention should be given to differentiate primary and metastatic ovarian tumors in breast cancer patients, often requiring immunohistochemical studies for this precise diagnosis.

\section{References}

1. Ernst M, Van de Poll-Franse L, Roukema J, Coebergh JW, van Gestel CM, et al. (2007) Trends in the prognosis of patients with primary metastatic breast cancer diagnosed between 1975 and 2002. Breast 16: 344-351. [Crossref]

2. Iwamoto N, Suzuki T, Tsukada K, Kunii Y, Haraguchi Y, et al. (2014) Metastasis of Breast Cancer to the Appendix Causing Acute Appendicitis: Report of a Case. Juntendo Medical Journal 2: 1-4.
3. Perrotin F, Marret H, Bouquin R, Fischer-Perrotin N, Lansac J, et al. (2001) Incidence, diagnosis and prognosis of ovarian metastasis in breast cancer. Gynecol Obstet Fertil 29: 308-315.

4. Lee YT, Hori JM (1971) Significance of ovarian metastasis in therapeutic oophorectomy for advanced breast cancer. Cancer 27: 1374-1378.

5. Connor SJ, Hanna GB, Frizelle FA, et al (1998) Retrospective clinicopathological analysis of appendiceal tumors from 7970 appendectomies. Dis Colon Rectu 41: 75-80.

6. Ratanarapee S, Nualyong C (2010) Acute appendicitis as primary symptom of prostatic adenocarcinoma: report of a case. J Med Assoc Thai 93: 1327-1331. [Crossref]

7. Wolf C, Friedl P, Obrist P, Ensinger C, Gritsch W (1999) Metastasis to the appendix: sonographic appearance and review of the literature. J Ultrasound Med 18: 23-25. [Crossref]

8. Petru E, Pickel H, Heydarfadai M, Lahousen M, Haas J, et al. (1992) Nongenital cancers metastatic to the ovary. Gynecol Oncol 44: 83-86. [Crossref]

9. Liu LY (1989) Metastatic cancer in the ovary--report of 57 cases. Zhonghua Zhong Liu Za Zhi 11: 464-467. [Crossref]

10. Gavriilidis P, Ananiadis A, Theodoulidis V, Toskas I, Barbanis S (2012) Ovarian metastasis from breast invasive ductal carcinoma. Hippokratia 16: 391. [Crossref]

11. Bigorie V, Morice P, Duvillard P, Antoine M, Cortez A, et al. (2010) Ovarian metastases from breast cancer: report of 29 cases. Cancer 116: 799-804. [Crossref]

12. Yoon WJ, Yoon YB, Kim YJ, Ryu JK, Kim YT (2010) Secondary appendiceal tumors: a review of 139 cases. Gut Liver 4: 351-356. [Crossref]

13. Eitan R, Gemignani ML, Venkatraman ES, Barakat RR, Abu-Rustum NR (2003) Breast cancer metastatic to abdomen and pelvis: role of surgical resection. Gynecol Oncol 90 397-401. [Crossref]

Copyright: (C2018 Araújo JL. This is an open-access article distributed under the terms of the Creative Commons Attribution License, which permits unrestricted use, distribution, and reproduction in any medium, provided the original author and source are credited. 